\title{
Investigating the kinetics of autotrophic denitrification with thiosulfate: modeling the denitritation mechanisms and the effect of the acclimation of SO-NR cultures to nitrite
}

\author{
Mabel Mora ${ }^{\mathrm{a}}$, Antonio David Dorado ${ }^{\mathrm{b}}$, Xavier Gamisans $^{\mathrm{b}}$, David Gabriel ${ }^{\mathrm{a}^{*}}$ \\ ${ }^{a}$ Department of Chemical Engineering, Escola d'Enginyeria, Universitat Autònoma de \\ Barcelona, 08193 Bellaterra, Spain, "e-mail: David.Gabriel@uab.cat, phone +34 935811587 \\ ${ }^{b}$ Department of Mining Engineering and Natural Resources, Universitat Politècnica de \\ Catalunya, Bases de Manresa 61-73, 08240 Manresa, Spain
}

\begin{abstract}
In this work the kinetics of a number of sulfide-oxidizing nitrate-reducing (SO-NR) cultures acclimated and not acclimated to nitrite were characterized. Anoxic respirometry coupled to kinetic modeling of respirometric profiles was the methodology used to study the two-step denitrification associated to thiosulfate oxidation. Autotrophic denitritation was initially studied in a non-acclimated SO-NR culture to confirm that nitrite reduction kinetics could be described through a Haldane-type equation. Afterwards, a kinetic model describing the two-step denitrification $\left(\mathrm{NO}_{3}{ }^{-} \rightarrow \mathrm{NO}_{2}{ }^{-} \rightarrow \mathrm{N}_{2}\right.$ ) was calibrated and validated through the estimation of several kinetic parameters from the fitting of experimental respirometric profiles obtained using either nitrate or nitrite as electron acceptors for both acclimated and non-acclimated biomass. The model proposed was a multi-substrate model that considered all the species implicated in the process as well as the stoichiometry associated particularly to the biomass studied in this work. A comparison between the kinetic parameters with the biomass acclimated and nonacclimated to nitrite revealed a 7-fold increase of the Haldane nitrite inhibition constant in the acclimated biomass with respect to the non-acclimated while the nitrite half-saturation constant and the maximum specific growth rate remained almost unchanged. The Fisher Information Matrix method was used to obtain the confidence intervals and also to evaluate the sensitivity and the identifiability in model calibration of each kinetic parameter estimated.
\end{abstract}

Keywords

Modeling; Autotrophic denitrification; Nitrite inhibition kinetics; Denitritation; SO-NR biomass acclimation; Fisher Information Matrix

\section{Introduction}

Different techniques have been developed to study the biodegradation characteristics of microbial cultures [1-2]. In particular, respirometry has been demonstrated as a powerful technique to gain insight on kinetics and stoichiometrics of biological processes [3-5] since biomass growth and substrate removal are directly associated with the electron acceptor consumption. Moreover, this technique allows obtaining biokinetic characteristics by modeling 
the respirometric profiles [6] as well as evaluating the inhibitory and/or toxic effects that a particular microbial population may suffer [7-8].

Kinetic characterization of autotrophic denitrifying biomass through respirometry has already been reported for suspended cultures obtained from wastewater treatment processes [9] and for SO-NR cultures obtained from anoxic desulfurizing biotrickling filters (BTF) [10]. However, in both cases the stoichiometric coefficients used and the kinetic parameters obtained were significantly different. This means that the use of some kinetic or stoichiometric data reported in literature for SO-NR biomass entails an inaccurate characterization of a specific biomass since the experimental conditions as well as the microbial diversity of biomass are hardly the same. In the case of immobilized biomass an additional bottle-neck is the estimation of biomass growth yield, biomass concentration and the active fraction of such microbial culture.

Some authors have used single-substrate kinetic models taking into account microbial growth rates associated only to a single pollutant biodegradation (Monod, Haldane and other kinetic equations) [11-12] to describe biological processes. A drawback of single-substrate kinetic models is the disability to describe the potential limitations of other species such as nutrients or the electron acceptor. Also, models based on single-substrates can hardly describe the formation of multiple end-products in complex biological processes such as biological denitrification and desulfurization processes [13]. For this reason, a multi-substrate model has to be proposed to describe the two-step denitrification since both electron acceptors (nitrate and nitrite) are implicated in the process. Moreover, the confidence intervals of the kinetic parameters are not commonly assessed even if they are as important as the estimation of the parameters themselves. The Fisher Information Matrix (FIM) method is a proven tool that accurately provides confidence intervals for kinetic parameters. This method is based on the calculation of the covariance matrix inverse, which is directly associated to the uncertainty of the model parameters estimated and the quantity and quality of the experimental data. Many authors have successfully used this mathematical method to evaluate the reliability of the parameters estimated both in wastewater and in polluted gas treatment processes [14-15]. The FIM method allows evaluating the sensitivities of the parameters and the quality of estimations.

Previous works have reported a methodology and an experimental setup of the anoxic respirometer used in this study. In addition, denitrification and thiosulfate oxidation rates of a SO-NR biomass from an anoxic biotrickling filter have been obtained as well as the stoichiometry of the two-step denitrification process has been solved [16-17]. However, kinetic equations and parameters that characterize the biological process have not been already reported. Consequently, the aim of the present study was to determine the kinetic parameters of the autotrophic denitrification mechanisms based on thiosulfate oxidation. The effect of culture acclimation to nitrite was also investigated and evaluated in this work through the changes in the kinetic parameters, which are directly related with the operating conditions of the reactor in which the biomass was grown and the history of the culture. For a reliable characterization of the kinetic model and parameters, the stoichiometric coefficients corresponding to the culture used in this study were those previously calculated through respirometric and titrimetric techniques in Mora et al. [17]. The acclimation of the biomass to nitrite was also assessed through kinetic parameters estimation and proposed to face nitrite inhibition problems in denitrifying reactors. 
2 Materials and methods

\subsection{Biomass}

The SO-NR biomass used in this work was obtained from an anoxic biogas desulfurizing biotrickling filter (BTF) at different operation times (175 d and $325 \mathrm{~d}$ of operation) [18]. The first sampling of the biomass from the BTF (175 d) was used to inoculate a $2.8 \mathrm{~L}$ continuous stirred tank reactor (CSTR). Once assessed the steady state, the study of denitritation kinetics was performed using the SO-NR biomass cultured in the CSTR (Operation A). This biomass was then discarded for further studies. The second sample of biomass obtained from the BTF (325 d) was cultured in the CSTR (Operation B) during 22 weeks, without nitrite accumulation, for assessing the two-step denitrification process. Later, the biomass was acclimated to nitrite 2 weeks before the end of the CSTR operation by decreasing stepwise the hydraulic retention time. A detailed description of the start-up and the continuous operation of the reactors are reported elsewhere [16].

\subsection{Respirometric profiles}

Two sets of respirometric tests were performed with the biomass cultured in the CSTR in order to evaluate the denitrification kinetics associated to thiosulfate oxidation (Table 1). During the first set of tests (Set A) nitrite was used as the electron acceptor in order to define the kinetic model describing denitritation. The second set (Set B) was performed to study the kinetics of the whole denitrification process and to assess the impact of biomass acclimation to nitrite. Biomass used in set A and B had the same origin (anoxic desulfurizing BTF) but were collected and cultivated in different periods of time (see section 2.1 - Operation A and B of the CSTR). Thus, impact of biomass acclimation was only assessed with biomass from set B before and after biomass acclimation to nitrite. Moreover, the use of thiosulfate allowed studying denitrification and nitrite acclimation clearly since the effects of many additional reduced sulfur compounds reactions were avoided. It would not be the case of sulfide since elemental sulfur can be formed as an intermediary product affecting consequently the denitrification rates [10].

\section{Here Table 1}

To obtain the respirometric profiles a certain volume of the biomass cultured in the CSTR was previously washed and poured into the respirometer. Subsequently, known pulses of thiosulfate, nitrate and nitrite were added to the respirometer after overcoming both the endogenous and the wake up phases. The continuous sampling of the system allowed monitoring the concentration of the species involved in the process, which enables the estimation of the corresponding kinetic parameters by modeling the respirometric profiles. The protocol used for biomass preparation prior to the anoxic respirometric tests as well as the set up of the respirometer and the procedure to obtain the respirometric profiles were previously optimized and properly described in Mora et al. [16].

\subsection{Chemical analysis}

Nitrite $\left(\mathrm{NO}_{2}{ }^{-}\right)$, sulfate $\left(\mathrm{SO}_{4}{ }^{2-}\right)$, nitrate $\left(\mathrm{NO}_{3}{ }^{-}\right)$and thiosulfate $\left(\mathrm{S}_{2} \mathrm{O}_{3}{ }^{2-}\right)$ concentrations were determined by ion chromatography with conductivity detection using a Dionex ICS-2000. The biomass concentration was determined according to Standard Methods [19]. The inorganic carbon concentration was measured with an OI Analytical TIC/TOC Analyzer (Model 1020A). The dissolved oxygen concentration and the $\mathrm{pH}$ were continuously monitored in-situ with 
sensors (CellOx ${ }^{\circledR} 325$ and SenTix ${ }^{\circledR} 82$ from WTW, respectively) connected to a bench-top multimeter (Inolab® Multi $740-$ WTW).

3 Development of the kinetic model

According to literature [20], nitrate reduction could be represented by a Monod-type equation while nitrite reduction can be described by Haldane-type kinetics. The accumulation of nitrite has also been reported as a kinetic affecting factor which could depend of the $\mathrm{pH}$ [21], the S/N ratio used [22-23], the competition for each electron acceptor (nitrite or nitrate) [24], etc. In this work none of these considerations were included in the kinetic model since in a previous study [16] it was observed that the factor affecting the progress of this process for a constant $\mathrm{pH}$ was the denitratation and denitritation kinetics, being this fact related with the biomass present in the culture.

\subsection{Stoichiometric coefficients}

Stoichiometric coefficients are necessary to properly characterize the kinetics of a microbial population since biomass growth yields have a strong influence on the relation between all the species implicated in the biological process. In this study the two-step denitrification stoichiometry used (Eqs. 1-2) was previously obtained by Mora et al. [17] through respirometric and titrimetric techniques for the same SO-NR biomass. From these coefficients the biomass growth yield related to substrate $\left(\mathrm{Y}_{\mathrm{X} / \mathrm{S}}\right)$ or any electron acceptor $\left(\mathrm{Y}_{\mathrm{X} / \mathrm{Nit}}\right.$ and $\left.\mathrm{Y}_{\mathrm{X} / \mathrm{N}}\right)$ and also the ratio between the species involved in the process can be calculated.

$$
\begin{gathered}
\mathrm{S}_{2} \mathrm{O}_{3}{ }^{2-}+2.626 \mathrm{NO}_{3}{ }^{-}+0.043 \mathrm{CO}_{2}+ \\
0.644 \mathrm{HCO}_{3}{ }^{-}+0.137 \mathrm{NH}_{4}{ }^{+}+0.631 \mathrm{H}_{2} \mathrm{O} \\
0.137 \mathrm{C}_{5} \mathrm{H}_{7} \mathrm{O}_{2} \mathrm{~N}+2.626 \mathrm{NO}_{2}{ }^{-}+1.494 \mathrm{H}^{+}+2 \mathrm{SO}_{4}{ }^{2-} \\
\mathrm{S}_{2} \mathrm{O}_{3}{ }^{2-}+2.070 \mathrm{NO}_{2}{ }^{-}+0.028 \mathrm{CO}_{2}+0.419 \mathrm{HCO}_{3}{ }^{-}+0.089 \mathrm{NH}_{4}{ }^{+}+0.400 \mathrm{H}^{+} \\
0.089 \mathrm{C}_{5} \mathrm{H}_{7} \mathrm{O}_{2} \mathrm{~N}+1.035 \mathrm{~N}_{2}+0.275 \mathrm{H}_{2} \mathrm{O}+2 \mathrm{SO}_{4}{ }^{2-}
\end{gathered}
$$

In previous works, in contrast with the present study, two-step denitrification have not been considered since nitrite was not observed during the respirometric assays [10].

\subsection{Modeling denitritation kinetics}

Nitrogen gas was the final product considered from nitrite reduction (Eq. 2). Several kinetic models have been previously proposed to describe denitritation [20, 25-28]. Often, accumulation of nitrite during denitrification and the consequent inhibition has been considered in such models [24, 29]. In this study the inhibition caused by nitrite on nitrite reduction was studied and described by a Haldane-type equation (Eq. 3) according to respirometric tests performed in Set A as discussed later.

$$
\mathrm{r}_{\text {degnitritation }}=\mathrm{r}_{\max } \frac{[\mathrm{Nit}]}{\mathrm{K}_{\mathrm{Nit}}+[\mathrm{Nit}]+\frac{[\mathrm{Nit}]^{2}}{\mathrm{~K}_{\mathrm{i}, \mathrm{Nit}}}}
$$


where $r_{\text {denitritation }}$ and $r_{\max }$ are the specific and the maximum specific denitritation rates (mg N$\mathrm{NO}_{2}^{-} \mathrm{g}^{-1} \mathrm{VSS} \mathrm{h}^{-1}$ ), respectively; [Nit] is the nitrite concentration ( $\mathrm{mg} \mathrm{N}-\mathrm{NO}_{2}{ }^{-} \mathrm{L}^{-1}$ ); $\mathrm{K}_{\mathrm{Nit}}$ is the half-saturation coefficient for nitrite $\left(\mathrm{mg} \mathrm{N}-\mathrm{NO}_{2}{ }^{-} \mathrm{L}^{-1}\right)$; and $\mathrm{K}_{\mathrm{i}, \mathrm{Nit}}$ is the nitrite inhibition constant (mg N-NO${ }_{2}^{-} \mathrm{L}^{-1}$ ).

In addition to the Haldane-type term for nitrite, the set of differential equations describing mass balances for nitrite and thiosulfate consumption, sulfate production and biomass growth (Eqs. 47) as well as a Monod-type term to describe thiosulfate oxidation were considered. No elemental sulfur accumulation was included in the model since, as previously referenced [16, $21,30]$, thiosulfate oxidation under anoxic conditions produced directly sulfate as the final product.

$$
\begin{aligned}
& \frac{\mathrm{d}[\mathrm{Nit}]}{\mathrm{dt}}=\stackrel{(4)}{-\frac{1}{\mathrm{Y}_{\mathrm{X}} / \mathrm{Nit}}} \cdot \mu_{\max , \mathrm{Nit}} \cdot \mathrm{X} \cdot \frac{[\mathrm{S}]}{\mathrm{K}_{\mathrm{S}}+[\mathrm{S}]} \cdot \frac{[\mathrm{Nit}]}{\mathrm{K}_{\mathrm{Nit}}+[\mathrm{Nit}]+\frac{[\mathrm{Nit}]^{2}}{\mathrm{~K}_{\mathrm{i}, \mathrm{Nit}}}} \\
& \frac{\mathrm{d}[\mathrm{S}]}{\mathrm{dt}}=\frac{(5)}{\mathrm{Y}_{\mathrm{Nit}}} \cdot \frac{\mathrm{d}[\mathrm{Nit}]}{\mathrm{dt}} \\
& \frac{(6) \mathrm{d})_{\mathrm{SS}]}^{\mathrm{dt}}}{\mathrm{dt}}=-\frac{\mathrm{d}[\mathrm{S}]}{\mathrm{dt}}
\end{aligned}
$$

$$
\frac{(\mathrm{d}[\mathrm{X}]}{\mathrm{dt}}=\mu_{\max , \mathrm{Nit}} \cdot \mathrm{X} \cdot \frac{[\mathrm{S}]}{\mathrm{K}_{\mathrm{S}}+[\mathrm{S}]} \cdot \frac{[\mathrm{Nit}]}{\mathrm{K}_{\mathrm{Nit}}+[\mathrm{Nit}]+\frac{[\mathrm{Nit}]^{2}}{\mathrm{~K}_{\mathrm{i}, \mathrm{Nit}}}}
$$

where $\mathrm{S}$ is the concentration of thiosulfate $\left(\mathrm{mg} \mathrm{S}-\mathrm{S}_{2} \mathrm{O}_{3}{ }^{2-} \mathrm{L}^{-1}\right)$; $\mathrm{SS}$ is the concentration of sulfate (mg S-SO ${ }_{4}^{2-} \mathrm{L}^{-1}$ ); $\mathrm{X}$ is the biomass concentration experimentally quantified as VSS concentration (mg VSS L ${ }^{-1}$ ); $\mu_{\text {max,Nit }}$ is the maximum specific growth rate of the biomass using nitrite as the electron acceptor $\left(\mathrm{h}^{-1}\right)$; $\mathrm{K}_{\mathrm{s}}$ is the half-saturation coefficient for thiosulfate (mg S$\mathrm{S}_{2} \mathrm{O}_{3}{ }^{2-} \mathrm{L}^{-1}$ ); and $\mathrm{Y}_{\mathrm{X} / \mathrm{Nit}}$ and $\mathrm{Y}_{\mathrm{Nit} / \mathrm{S}}$ are the stoichiometric biomass growth yield related to nitrite (mg VSS $\mathrm{mg} \mathrm{N}^{-1}$ ) and the nitrite to thiosulfate ratio ( $\mathrm{mg} \mathrm{N} \mathrm{mg} \mathrm{S}^{-1}$ ), respectively.

\subsection{Modeling the two-step denitrification}

The denitrification process has been previously characterized through several kinetic equations combining non-competitive inhibition between denitrification intermediates and simple Monod equations [20, 27]. In this work, the two-step denitrification mechanism associated to thiosulfate oxidation (experimental tests corresponding to set B) was modeled considering rate limitation caused by nitrate and inhibition caused by nitrite over denitritation. Moreover, no competition between the electron acceptors has been taken into account neither the influence of the initial S$\mathrm{S}_{2} \mathrm{O}_{3}{ }^{2-} / \mathrm{N}_{-} \mathrm{NO}_{3}{ }^{-}$ratio on denitrification mechanisms since in previous results none of these phenomena were observed [16]. Simulation of the respirometric profiles was performed by taking into account equations 4 to 7 and the differential equations corresponding to nitrate consumption (Eqs. 8-10).

$\frac{\mathrm{d}[\mathrm{N}]}{\mathrm{q}] \mathrm{t}}=-\frac{1}{\mathrm{Y}_{\mathrm{X} / \mathrm{N}}} \cdot \mu_{\max , \mathrm{N}} \cdot \mathrm{X} \cdot \frac{[\mathrm{S}]}{\mathrm{K}_{\mathrm{S}}+[\mathrm{S}]} \cdot \frac{[\mathrm{N}]}{\mathrm{K}_{\mathrm{N}}+[\mathrm{N}]}$ 


$$
\begin{aligned}
& \frac{\mathrm{d}[\mathrm{S}]}{\mathrm{dt}}=\frac{1}{\mathrm{Y}_{\mathrm{Nit} / \mathrm{S}}} \cdot \frac{\mathrm{d}[\mathrm{Nit}]}{\mathrm{dt}}+\frac{1}{\mathrm{Y}_{\mathrm{N} / \mathrm{S}}} \cdot \frac{\mathrm{d}[\mathrm{N}]}{\mathrm{dt}} \\
& \frac{\mathrm{d}[\mathrm{X}]}{\mathrm{dt}}=\mu_{\max , \mathrm{N}} \cdot \mathrm{X} \cdot \frac{[\mathrm{S}]}{\mathrm{K}_{\mathrm{S}}+[\mathrm{S}]} \cdot \frac{[\mathrm{N}]}{\mathrm{K}_{\mathrm{N}}+[\mathrm{N}]}
\end{aligned}
$$

where $[\mathrm{N}]$ is the concentration of nitrate $\left(\mathrm{mg} \mathrm{N}-\mathrm{NO}_{3}{ }^{-} \mathrm{L}^{-1}\right), \mu_{\max , \mathrm{N}}$ is the maximum specific growth rate of the biomass using nitrate as the electron acceptor $\left(h^{-1}\right), K_{N}$ is the half-saturation coefficient for nitrate (mg N-NO${ }_{3}^{-} \mathrm{L}^{-1}$ ) and $\mathrm{Y}_{\mathrm{X} / \mathrm{N}}$ and $\mathrm{Y}_{\mathrm{N} / \mathrm{S}}$ are the stoichiometric biomass growth

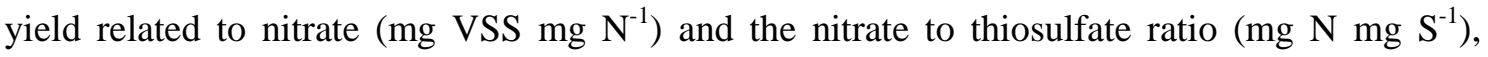
respectively.

\subsection{Parameter estimation}

Maximum specific growth rates, half-saturation constants and inhibition constants were estimated as well as the respirometric profiles were simulated by means of MATLAB 7.7 (Mathworks, Natik, MA). The differential equations presented above (Eqs. 4-10) were solved using a variable step Runge-Kutta method and the parameter estimation was carried out by using the Nelder-Mead Simplex search method algorithm (unconstrained non-linear optimization). The functions employed for the numerical solution were ode45 and fminsearch, respectively. In this case, the fitting of the experimental data to model predictions uniquely considers the electron acceptors (nitrite and nitrate) and it is based on seeking the minimum value of the objective function (F) (Eq. 11). This function is defined as the norm of the differences between the predicted values of the mathematical model and the experimental data:

$\stackrel{(11)}{\sum_{\mathrm{i}=1}^{\mathrm{n}}\left[\mathrm{y}_{\mathrm{exp}, \mathrm{i}}-\mathrm{y}_{\theta, \mathrm{i}}\right]^{2}}$

where $\mathrm{n}$ is the number of experimental data, $\mathrm{y}_{\theta, \mathrm{i}}$ is the predicted value with the kinetic parameters to estimate $(\theta)$ and $y_{\text {exp, }}$ is the value experimentally measured.

\subsection{Fisher Information Matrix}

The confidence intervals of the estimated parameters were assessed through the Fisher Information Matrix mathematical method. The FIM summarizes the quantity and quality of information obtained in each experiment because it considers the output sensitivity functions and the measurement errors of an experimental data (i.e. accuracy of an experiment). When white measurement noise (i.e. independent and normally distributed with zero mean) and no model mismatch, no data autocorrelation and uncorrelated errors can be assumed, the inverse of the FIM provides the lower bound of the parameter estimation error covariance matrix, which can be used for assessing the estimation uncertainty of the optimal estimated parameters. Moreover, since output sensitivities of parameters are calculated using a model, the FIM also depends on the model structure [14-15, 31-32]. 
4 Results and discussion

\subsection{Modeling of denitritation kinetics}

From the experiments performed in Set A with a SO-NR culture not-acclimated to nitrite (Table 1) three respirometric profiles were obtained at different initial nitrite concentrations (Fig. 1, ac). From the slopes of nitrite concentration profiles many nitrite uptake rates (NitUR) were calculated for different nitrite concentrations and plotted as a function of the nitrite concentration in Fig. 1d. A Haldane-type equation (Eq. 3) was properly fitted to the experimental kinetic profile (Fig. 1d) to estimate $r_{\max }, K_{\mathrm{Nit}}$ and $K_{\mathrm{i}, \mathrm{Nit}}$ (Table 2). The $\mu_{\max , \mathrm{Nit}}$ (Eq. 4) was also calibrated using the respirometric test A-2 (Fig. 1b) since a sensitivity analysis showed that the predicted nitrite concentration was more influenced by variations of $\mu_{\max , \text { Nit }}$ in test A-2 than in tests A-1 and A-3. The half-saturation coefficient for thiosulfate $\left(\mathrm{K}_{\mathrm{S}}\right)$ was not calibrated since the concentration used almost in all respirometric tests was always above 120 mg S- $\mathrm{S}_{2} \mathrm{O}_{3}{ }^{2-} \mathrm{L}^{-1}$, which is much higher than the $\mathrm{K}_{\mathrm{S}}$ reported in literature. A $\mathrm{K}_{\mathrm{S}}$ of $32.4 \mathrm{mg} \mathrm{S}-$ $\mathrm{S}_{2} \mathrm{O}_{3}{ }^{2-} \mathrm{L}^{-1}$ reported in Artiga et al. [33] for a thiosulfate-oxidizing nitrate-reducing culture was used. As shown in Fig. 1 (a, c), the denitritation profiles, as well as the corresponding thiosulfate oxidation associated, were satisfactorily described through the Haldane-type equation previously calibrated with the kinetic profile (Fig. 1d) and the test A-2 (Fig. 1b). Literature regarding kinetics of bacterial growth with thiosulfate using nitrite as the electron acceptor is very limited and prior to the present work no data for biokinetic parameters were reported. However, Fajardo et al. [34] obtained a maximum specific uptake rate of $3.7 \mathrm{mg} \mathrm{N}$ $\mathrm{NO}_{2}^{-} \mathrm{g} \mathrm{VSS}^{-1} \mathrm{~h}^{-1}$ using nitrite as electron acceptor with a sulfide-oxidizing biomass cultivated in a sequencing batch reactor (SBR). This rate was almost 15 times lower than that obtained in the present work (54.1 mg N g VSS ${ }^{-1} \mathrm{~h}^{-1}$ ) indicating that the cultures cannot be compared and that specific tests must be performed for each microbial culture to properly assess its kinetic parameters.

\section{Here Fig. 1}

Table 2 shows the confidence intervals in the estimation of each parameter obtained through the FIM method. As can be observed, a maximum confidence interval of $0.34 \%$ was determined for the half-saturation constant $\left(\mathrm{K}_{\mathrm{Nit}}\right)$ indicating that the high sensitivity of all kinetic parameters at these concentration ranges guarantees the parameter identifiability during model calibration (i.e. the possibility in giving an unique value of each parameter in the model). Such low confidence intervals indicate both the use of an accurate measurement technique as well as a correct experimental design that satisfactorily described the experimental behavior with the kinetic model proposed.

\section{Here Table 2}

The maximum specific growth rate estimated $\left(\mu_{\text {max,Nit }}\right)$ was slightly lower than the dilution rate set in the reactor $\left(0.018 \mathrm{~h}^{-1}\right)$. This result reveals that the utilization of nitrite as electron acceptor in the CSTR would have caused the wash out of biomass.

4.2 Calibration of the two-step denitrification kinetic model.

Experiments corresponding to Set B (Table 1) were performed with the biomass cultured in the CSTR during Operation B. In Set B, tests with biomass acclimated and non-acclimated to nitrite 
were carried out to completely calibrate the two-step denitrification model and to determine the influence of biomass acclimation to nitrite over SO-NR thiosulfate-based denitrification kinetics. Calibration of the two-step denitrification kinetic model was performed with tests B-1 and B-2 with acclimated biomass. Once kinetics corresponding to nitrate reduction had been obtained, the denitritation parameters were estimated with experimental data from test B-3 for the non-acclimated biomass.

\subsubsection{Biomass acclimated to nitrite}

Similarly to the non-acclimated biomass tests in set A, kinetic parameters related to denitritation ( $\mu_{\text {max,Nit }}, K_{N i t}$ and $K_{i, N i t}$ ) with the biomass acclimated to nitrite were estimated (Table 3 ) from respirometric test B-1 using a Haldane-type equation (Eq. 3) which was confirmed above (section 4.1) that is adequate to describe denitritation. Fig. 2a shows that the model satisfactorily fits the experimental data over again even if larger confidence intervals than those obtained through the nitrite uptake rate profile for the non-acclimated biomass (section 4.1) were obtained. In this case a nitrite uptake rate profile could not be performed since no experimental data was available at nitrite inhibiting concentrations. Still results were considered satisfactory since none of the confidence intervals exceed a $5 \%$ of deviation. As expected, a higher confidence interval was found for $\mathrm{K}_{\mathrm{Nit}}$ since the nitrite concentration range in the tests was not the most suitable for its estimation. Nonetheless, results indicate that the compilation of several nitrite uptake rates from respirometric tests performed under different conditions is very helpful to properly fit a kinetic equation to the experimental data even if inhibition concentrations were not achieved. It is worth mentioning that a fair comparison of inhibition constants from Table 2 and Table 3 for the non-acclimated and acclimated biomass, respectively, was not possible since both microbial populations were different as indicated by the differences in their maximum specific growth rates.

\section{Here Table 3}

Regarding the calibration of nitrate reduction, kinetic parameters previously estimated from test B-2 were used to estimate $\mu_{\max , \mathrm{N}}, \mathrm{K}_{\mathrm{N}}$ and $\mathrm{K}_{\mathrm{s}}$. A K $\mathrm{K}_{\mathrm{S}}$ of $16.1 \pm 0.04 \mathrm{mg} \mathrm{S}-\mathrm{S}_{2} \mathrm{O}_{3}{ }^{2-} \mathrm{L}^{-1}$ was obtained, which was slightly different from that reported by Artiga et al. [33], indicating that the use of the parameter reported in literature for concentrations up to $120 \mathrm{mg} \mathrm{S}-\mathrm{S}_{2} \mathrm{O}_{3}{ }^{2-} \mathrm{L}^{-1}$ lead to more than $10 \%$ error in model predictions. The $\mu_{\max , \mathrm{N}}$ obtained $\left(0.0269 \pm 0.0001 \mathrm{~h}^{-1}\right)$ was very low compared with those reported for SO-NR biomass $\left(0.05-0.2 \mathrm{~h}^{-1}\right)$ [9-10, 35] probably because the biomass was obtained from a desulfurizing biotrickling filter. In such biofilm-based reactors, biomass is not naturally selected because of its growth rate but for the resistance and acclimation to specific operating conditions. The estimated $\mathrm{K}_{\mathrm{N}}$ indicates that nitrate would not be limiting the reaction at concentrations above $20 \mathrm{mg} \mathrm{N}-\mathrm{NO}_{3}^{-} \mathrm{L}^{-1}$ since this value allows reaching a $95 \%$ of the maximum reaction rate.

\section{Here Fig. 2}

Biomass acclimated to nitrite presented high denitritation rates since no accumulation of nitrite was observed in test B-2 (Fig. 2b). This fact avoids the inhibition caused by nitrite over thiosulfate oxidation under anoxic conditions and allows using high concentrations of nitrate in the biofilter without limiting the reaction rate. Therefore, biomass acclimation to nitrite can be favorably implemented in those processes in which denitritation is lower than denitratation causing nitrite accumulation and, consequently, an efficiency drop due to an inhibiting situation. 


\subsubsection{Biomass non-acclimated to nitrite}

Test B-3 (Fig.3) was performed with the same biomass used in tests B-1 and B-2 previously to the acclimation period to nitrite to uniquely assess the effect of nitrite acclimation in the estimation of the kinetic parameters of the denitritation mechanism. In this test (B-3), the denitritation model was calibrated by using the kinetic parameters corresponding to nitrate reduction obtained from the model calibration performed with test B-2. These parameters were used on the basis that nitrite acclimation affected uniquely to denitritation. As shown in Fig. 3, the calibrated kinetic model accurately described the experimental data corresponding to the two-step denitrification associated to thiosulfate oxidation. The biomass concentration profile was also predicted considering the growth yields obtained from the stoichiometry $\left(\mathrm{Y}_{\mathrm{X} / \mathrm{N}}\right.$ and $\left.\mathrm{Y}_{\mathrm{x} / \mathrm{Nit}}\right)$ and also a $100 \%$ of active biomass. As can be observed, the biomass concentration increased by $15 \%$ from its initial value. This biomass growth was considered to not overestimate the kinetic parameters. In Table 4 the estimated parameters and the corresponding confidence intervals are presented for the non-acclimated biomass.

\section{Here Fig. 3}

\section{Here Table 4}

\subsubsection{Effect of nitrite acclimation on kinetic parameters}

Comparison of the parameters in Table 3 and Table 4 indicates that biomass acclimation to nitrite improved characteristics related to the nitrite inhibition effect. In terms of growth both microbial cultures presented a similar maximum specific growth rate indicating that microbial cultures did not changed before and after acclimation. However, the inhibition constant for the acclimated biomass $\left(75.2 \pm 2.5 \mathrm{mg} \mathrm{N} \mathrm{L}^{-1}\right)$ was almost 7-fold that obtained with the nonacclimated biomass $\left(11.5 \pm 0.4 \mathrm{mg} \mathrm{N} \mathrm{^{-1 }}\right)$ meaning that the acclimated biomass shows lower degree of nitrite inhibition. Concerning biomass affinity to nitrite, similar half-saturation constants were estimated although the $\mathrm{K}_{\mathrm{Nit}}$ was slightly lower for the acclimated biomass. This result was somehow expected since the biomass was acclimated by reducing stepwise the dilution rate being accumulated the nitrite until reaching concentrations of about $150 \mathrm{mg} \mathrm{N}$ $\mathrm{NO}_{2}^{-} \mathrm{L}^{-1}$. In this sense the biomass was forced to be capable of growing at this elevated concentration being the influence of the half-saturation constant negligible and, consequently, not affected by the acclimation. This results lead to confirm that the culture presented a high resistance to nitrite accumulation, since the $\mu_{\max , \text { it }}$ was almost maintained, but also a high resilience [36] since the concentration of nitrite decreased after reaching the maximum concentration although maintaining a lower HRT (data not shown).

As expected, the parameters obtained in set B were different from those previously obtained in set A (section 4.1) since the biomass was obtained at different operation times of the BTF. The kinetic parameters reported in Mora et al. [10] using SO-NR biomass from the same anoxic desulfurizing BTF were also different from those estimated in this study since the biomass was obtained 1 year later. These results remark the importance of the biomass history and the effect of operating conditions fluctuations over culture characteristics and kinetic behavior.

Finally, in this calibration the uncertainties for all the parameters were slightly higher than those obtained in previous calibrations for denitritation and two-step denitrification biomass acclimated to nitrite (sections 4.1 and 4.2.1) but specially the uncertainty associated to $\mathrm{K}_{\mathrm{Nit}}$ and $\mathrm{K}_{\mathrm{i}, \text { Nit. }}$. It is worthy to mention that the confidence intervals assessed through the FIM method could be reduced by increasing the quantity of experimental data measured, which can be 
considered relatively low in these experiments. Even so, all kinetic parameters were identifiable on the basis of respiration tests indicating that the conditions selected to calibrate the model were especially suitable.

\section{Conclusions}

From the results obtained in this work it can be concluded that the two-step denitrification associated to thiosulfate oxidation was successfully described with the kinetic model proposed and calibrated herein, which has not been previously reported in other studies. The calibration of the model allowed the experimental data fitting as well as the prediction of the biomass growth associated, which was necessary to properly estimate the kinetic parameters.

Denitritation was satisfactorily described through a Haldane equation with the SO-NR cultures acclimated and non-acclimated to nitrite. The effect of nitrite acclimation was also evaluated and it was confirmed that the biomass presented high resistance and resilience to nitrite concentrations up to $150 \mathrm{mg} \mathrm{N} \mathrm{L}^{-1}$.

Two-step denitrification could be correctly described by using the stoichiometric coefficients corresponding particularly to the biomass used in this work. Terms related to a non-competitive inhibition between nitrate and nitrite were excluded since this behavior was not observed with the SO-NR culture studied. In addition, the kinetic parameters estimated presented a high sensitivity to model predictions since small confidence intervals were assessed with the FIM method.

Results obtained in this work provide complementary information to research related with the simultaneous desulfurization and denitrification required to treat waste liquid effluents, as thiosulfate or nitrate containing wastewaters with low organic carbon content. This would be the case of SANI process intermediate effluents, which may contain high concentrations of thiosulfate as a result of a sulfate/sulfite reduction [37]. The presence of thiosulfate has even been reported as an advantage for the subsequent autotrophic denitrification integrated in the abovementioned SANI process [38]. Another interesting applicability of this study could be the nitrate removal of fish cannery effluents treated by anaerobic digestion [34]. In addition, this research provides useful knowledge about both the biochemical processes of SO-NR microbial populations and the mathematical modeling of biological denitrification processes, which is essential to improve the design and operation of anoxic desulfurizing BTFs.

\section{Acknowledgements}

The Spanish government (MEC) provided financial support through the Project CICYT CTM2009-14338-C03/FEDER and CTM2012-37927-C03/FEDER. The Department of Chemical Engineering at UAB (Universitat Autònoma de Barcelona) is a unit of Biochemical Engineering of the Xarxa de Referència en Biotecnologia de Catalunya (XRB), Generalitat de Catalunya. The authors would like also acknowledge to Javier Guerrero and the Group of Biological and Enzymatic Reactors from the University of Cádiz the collaboration in this work. Mabel Mora gratefully acknowledges an FPI predoctoral scholarship from Ministerio de Economía y Competitividad (Spain) 
[1] B.S. Moraes, T.S.O. Souza, E. Foresti, Characterization and kinetics of sulfide-oxidizing autotrophic denitrification in batch reactors containing suspended and immobilized cells, Water Sci. Technol., 64 (2011) 731-738.

[2] A. Dapena-Mora, J.L. Campos, A. Mosquera-Corral, R. Mendez, Development and application of an Anammox activity test based on gas production, European Symposium on Environmental Biotechnology, Eseb 2004, (2004) 649-652.

[3] I. Jubany, J.A. Baeza, J. Carrera, J. Lafuente, Respirometric calibration and validation of a biological nitrite oxidation model including biomass growth and substrate inhibition, Water Res., 39 (2005) 4574-4584.

[4] R.G. Riefler, D.P. Ahlfeld, B.F. Smets, Respirometric assay for biofilm kinetics estimation: Parameter identifiability and retrievability, Biotechnol. and Bioeng., 57 (1998) 35-45.

[5] G. Munz, R. Gori, G. Mori, C. Lubello, Monitoring biological sulphide oxidation processes using combined respirometric and titrimetric techniques, Chemosphere, 76 (2009) 644-650.

[6] H. Spanjers, P. Vanrolleghem, Respirometry as a tool for rapid characterization of wastewater and activated sludge, Water Sci. Technol., 31 (1995) 105-114.

[7] E.U. Cokgor, G. Insel, E. Aydin, D. Orhon, Respirometric evaluation of a mixture of organic chemicals with different biodegradation kinetics, J. Hazard. Mater., 161 (2009) 35-41.

[8] T.G. Ellis, D.S. Barbeau, B.F. Smets, C.P.L. Grady, Respirometric technique for determination of extant kinetic parameters describing biodegradation, Water Environ. Res., 68 (1996) 917-926.

[9] S.E. Oh, K.S. Kim, H.C. Choi, J. Cho, I.S. Kim, Kinetics and physiological characteristics of autotrophic dentrification by denitrifying sulfur bacteria, Water Sci. Technol., 42 (2000) 59-68.

[10] M. Mora, M. Fernández, J. Gómez, D. Cantero, J. Lafuente, X. Gamisans, D. Gabriel, Kinetic and stoichiometric characterization of anoxic sulfide oxidation by SO-NR mixed cultures from anoxic biotrickling filters, Appl. Microbiol. Biotechnol., (2014) 1-11.

[11] J. Cai, P. Zheng, M. Qaisar, Y. Xing, Kinetic characteristics of biological simultaneous anaerobic sulfide and nitrite removal, Desalin. and Water Treat., (2013) 1-8.

[12] M.C. Delhomenie, J. Nikiema, L. Bibeau, M. Heitz, A new method to determine the microbial kinetic parameters in biological air filters, Chem. Eng. Sci., 63 (2008) 41264134.

[13] J.B.M. Klok, M. de Graaff, P.L.F. van den Bosch, N.C. Boelee, K.J. Keesman, A.J.H. Janssen, A physiologically based kinetic model for bacterial sulfide oxidation, Water Res., 47 (2013) 483-492.

[14] A.D. Dorado, G. Baquerizo, J.P. Maestre, X. Gamisans, D. Gabriel, J. Lafuente, Modeling of a bacterial and fungal biofilter applied to toluene abatement: Kinetic parameters estimation and model validation, Chem. Eng. J., 140 (2008) 52-61.

[15] A. Guisasola, J.A. Baeza, J. Carrera, G. Sin, P.A. Vanrolleghem, J. Lafuente, The influence of experimental data quality and quantity on parameter estimation accuracy: Andrews inhibition model as a case study, Educ. Chem. Eng., 1 (2006) 139-145.

[16] M. Mora, A. Guisasola, X. Gamisans, D. Gabriel, Examining thiosulfate-driven autotrophic denitrification through respirometry, Chemosphere, 113 (2014) 1-8.

[17] M. Mora, L.R. López, X. Gamisans, D. Gabriel, Coupling respirometry and titrimetry for the characterization of the biological activity of a SO-NR consortium, Chem. Eng. J., 251 (2014) 111-115.

[18] A.M. Montebello, M. Fernandez, F. Almenglo, M. Ramirez, D. Cantero, M. Baeza, D. Gabriel, Simultaneous methylmercaptan and hydrogen sulfide removal in the desulfurization of biogas in aerobic and anoxic biotrickling filters, Chem. Eng. J., 200 (2012) 237-246.

[19] APHA-AWWA-WPCF, Standard Methods for the Examination of Water and Wastewater, 21st ed. American Public Health Association, Washington, 2005

[20] O. Soto, E. Aspe, M. Roeckel, Kinetics of cross-inhibited denitrification of a high load wastewater, Enzyme Microbiol. Technol., 40 (2007) 1627-1634. 
[21] H. Furumai, H. Tagui, K. Fujita, Effects of $\mathrm{pH}$ and alkalinity on sulfur denitrification in a biological granular filter, Water Sci. and Technol., 34 (1996) 355-362.

[22] I. Manconi, A. Carucci, P. Lens, Combined removal of sulfur compounds and nitrate by autotrophic denitrification in bioaugmented activated sludge system, Biotechnol. Bioeng., 98 (2007) 551-560.

[23] S. An, K. Tang, M. Nemati, Simultaneous biodesulphurization and denitrification using an oil reservoir microbial culture: effects of sulphide loading rate and sulphide to nitrate loading ratio, Water Res., 44 (2010) 1531-1541.

[24] B. Krishnakumar, V.B. Manilal, Bacterial oxidation of sulphide under denitrifying conditions, Biotechnol. Lett., 21 (1999) 437-440.

[25] C. Estuardo, M.C. Marti, C. Huilinir, E.A. Lillo, M.R. von Bennewitz, Improvement of nitrate and nitrite reduction rates prediction, Electron. J. Biotechnol., 11 (2008).

[26] J.S. Almeida, M.A.M. Reis, M.J.T. Carrondo, Competition between nitrate and nitrite reduction in denitrification by Pseudomonas Fluorescens, Biotechnol. Bioeng., 46 (1995) 476-484.

[27] D. Wild, R. Vonschulthess, W. Gujer, Structured modeling of denitrification intermediates, Water Sci. Technol., 31 (1995) 45-54.

[28] C. Glass, J. Silverstein, J. Oh, Inhibition of denitrification in activated sludge by nitrite, Water Environ. Res., 69 (1997) 1086-1093.

[29] M.R. Betlach, J.M. Tiedje, Kinetic explanation for accumulation of nitrite, nitric-oxide, and nitrous-oxide during bacterial denitrification, Appl. Environ. Microbiol., 42 (1981) 10741084.

[30] J.L. Campos, S. Carvalho, R. Portela, A. Mosquera-Corral, R. Mendez, Kinetics of denitrification using sulphur compounds: Effects of $\mathrm{S} / \mathrm{N}$ ratio, endogenous and exogenous compounds, Biores. Technol., 99 (2008) 1293-1299.

[31] R.K. Mehra, Optimal Input Signals for Parameter Estimation in Dynamic Systems Survey and New Results, IEEE Trans. Autom. Control, Ac19 (1974) 753-768.

[32] D. Dochain, P.A. Vanrolleghem, Dynamical Modelling and Estimation in Wastewater Treatment Processes, IWA publishing, 2001.

[33] P. Artiga, F. Gonzalez, A. Mosquera-Corral, J.L. Campos, J.M. Garrido, E. Ficara, R. Mendez, Multiple analysis reprogrammable titration analyser for the kinetic characterization of nitrifying and autotrophic denitrifying biomass, Biochem. Eng. J., 26 (2005) 176-183.

[34] C. Fajardo, M. Mora, I. Fernandez, A. Mosquera-Corral, J.L. Campos, R. Mendez, Cross effect of temperature, $\mathrm{pH}$ and free ammonia on autotrophic denitrification process with sulphide as electron donor, Chemosphere, 97 (2014) 10-15.

[35] G. Claus, H.J. Kutzner, Physiology and kinetics of autotrophic denitrification by Thiobacillus Denitrificans, Appl. Microbiol. Biotechnol., 22 (1985) 283-288.

[36] L. Cabrol, L. Malhautier, F. Poly, X. Le Roux, A.S. Lepeuple, J.L. Fanlo, Resistance and resilience of removal efficiency and bacterial community structure of gas biofilters exposed to repeated shock loads, Biores. Technol., 123 (2012) 548-557.

[37] F. Jiang, L. Zhang, G.L. Peng, S.Y. Liang, J. Qian, L. Wei, G.H. Chen, A novel approach to realize SANI process in freshwater sewage treatment - Use of wet flue gas desulfurization waste streams as sulfur source, Water Res., 47 (2013) 5773-5782.

[38] J. Qian, F. Jiang, H.K. Chui, M.C.M. van Loosdrecht, G.H. Chen, Industrial flue gas desulfurization waste may offer an opportunitty to facilitate SANI application for significant sludge minimization in freshwater wastewater treatment, Water Sci. Technol., 67 (2013) 2822-2826. 